\title{
LA TEOLOGÍA DE LA LIBERACIÓN DEL ECUADOR LÍDERES, PRINCIPIOS Y ESTILO DE IGLESIAS
}

\author{
Boris Tobar Solano'
}

\section{RESUMEN}

Este artículo aborda la historia de la teología de la liberación en Ecuador, vivida y testimoniada por pastores emblemáticos de las comunidades eclesiales, que con su labor dignificaron la vida de los indígenas, de los pobres, de las mujeres y del conjunto de las personas que participan de la vida comunitaria. En primer lugar se aproxima a la práctica de Leonidas Proaño quien desde su opción por los indios, construyó una teología de la solidaridad y dignificación de la persona. En segundo lugar recuerda la práctica de pastores como Mons. Luna Tobar, Gonzalo López y Padre José Carollo, quienes hicieron y vivieron su opción por los pobres desde los principios de solidaridad-liberación. En tercer lugar, visibiliza la práctica pastoral de colectivos eclesiales, que en tiempos de globalización, se convierten en sujetos históricos apasionados por la vida, y por la diversidad como mujeres, ecologistas y pueblos profundos, que desde su fe caminan comprometidos en el cuidado de la vida.

Palabras clave: teología, indios, pobres, iglesia, CEB.

\section{ABSTRACT}

This article approaches the history of Liberation Theology in Ecuador, lived and witnessed by emblematic ministers of ecclesial communities, whose work dignified the life of indigenous people, the poor, the women and everyone who takes part of community life. First, it remarks the practice of Mgr. Leonidas Proaño, who from his "option for the indians" built a theology of solidarity and personal dignification. Then, it recalls the practice of other ministers as Mgr. Luna Tobar, Gonzalo Lopez and Father Jose Carollo, who took and lived

${ }^{1}$ Pontificia Universidad Católica del Ecuador, Escuela de Trabajo Social, Quito, Ecuador (cbtobar@puce.edu.ec). 
their "preferential option for the poor" from the principles: solidarity-liberation. In the end, it shows the pastoral work of the base ecclesial communities, which in times of globalization, became historical subjects passionate about life and di-

En la voz de Francisco, la Iglesia Católica ha retomado la palabra profética que denuncia la injusticia de un sistema idolátrico que solo piensa en la reproducción del capital y olvida el cuidado de la vida, por eso, con su palabra, estilo de vida y otros signos como la canonización de Mons. Óscar Romero, el Papa Francisco de hecho ha rehabilitado filones de la teología de la liberación nacida en suelo latinoamericano. En este marco, el artículo aborda la historia de la teología liberadora vivida y testimoniada por comunidades comprometidas de la Iglesia del Ecuador para recuperar sus principios y convicciones, y desde allí, entrar en sintonía con la espiritualidad que anima a Francisco, primer Papa latinoamericano.

La teología de la liberación del Ecuador (TLE), en primera instancia, está ligada a la figura de Leonidas Proaño Villalba, obispo de la provincia de Chimborazo, lugar geográfico que hacia finales de los años 50 registra la mayor población indígena del país y al mismo tiempo la mayor explotación y miseria que grupo humano puede haber vivido versity, as women, environmental activists and "deep people", walking committed by their faith to care life.

Key words: theology, indian, poor, church. CEB.

en Ecuador. Proaño, gracias a la formación de una iglesia viva, comunitaria, logró un giro fundamental en la vida de las comunidades indígenas que hoy se han convertido en un sujeto histórico con palabra propia. Luego, la TLE, se enriquece con la práctica y testimonio de un puñado de obispos, como Alberto Luna Tobar, Gonzalo López Marañón, muchos religiosos y religiosas, pero sobre todo del trabajo de laicas y laicos de las Comunidades Eclesiales de Base (CEB) campesinas y urbano-marginales que trabajan por la defensa de su dignidad, la gestión comunitaria y una vivencia de fe comprometida con la transformación social. Los inicios de los noventa con todo el peso del contexto mundial "desencantador" la TL sufrió un nuevo impulso en Ecuador, ligado a las palabra resistencia, esperanza, vida para los pueblos, para la Pachamama, para las mujeres y otros actores emergentes, que desde la práctica, reflexión y sueños que aporta la teología se abren a seguir a Jesús el predicador del Reino, al Dios de la vida y a Espíritu que aletea en los diversos colectivos eclesiales que asumen el reto de ser y construir comuni- 
dades de vida, con rostro femenino, ecológico, migrante, étnico, generacional, incluyente y diverso.

Además, la teología ecuatoriana no es una reflexión académica, sistematizada, organizada por tratados. Es una teología pastoral nacida de la palabra y escritos cercanos de pastores, teólogos, laicos y laicas que tienen como interlocutor a comunidades concretas, y como marco referencia la realidad de negación, pobreza y exclusión que atraviesa la historia del Ecuador contemporáneo y que la iglesia liberadora ha sabido acompañar y sigue acompañando.

Este artículo desarrolla tres momentos claves de la práctica y reflexión de la TL en el Ecuador, cada uno se corresponde con una determinada época de la realidad ecuatoriana: el primero de opción por los indios, que se centra en la Iglesia del Chimborazo y en el trabajo pastoral de Leonidas Proaño, cuya práctica eclesial se condensa en la expresión "hay que caminar con los dos pies, uno en la iglesia y otro en la organización social"; es una teología que se mueve por los principios solidaridad, dignificación- organización. El segundo momento de práctica de la opción por los pobres, que surge de las CEB, sobre todo en las barriadas populares de la ciudad y el campo, movidos por los principios de solidaridad-liberación. El tercer momento de diversidad de sujetos históricos articulado por principios como resistencia, pasión por la vida, y diversidad que se descubre en la práctica de muchas organizaciones eclesiales y sociales, que desde la acción y reflexión comunitaria creyente, profundizan la identidad de sus luchas orientadas al mejoramiento de la calidad de vida de diversos actores sociales y el cuidado de la gran casa.

En cada momento histórico se presenta el contexto socio-económico, el ambiente eclesial, los principios y características de la teología y prácticas pastorales, la tensión con la iglesia jerárquica, los símbolos bíblico-teológicos inspiradores, la pedagogía popular que acompaña el trabajo evangelizador, la organizaciones eclesiales y sociales que nacen y se inspiran en la $T L$, y por último, se evalúa los logros y retos de cada momento.

\section{LA TEOLOGÍA DE LA SOLIDARIDAD, ILA OPCIÓN POR LOS INDIOS!}

La teología liberadora surgió en Ecuador en un contexto de vigencia de una institución llamada hacienda, organización económica productiva semi- feudal fundada en el trabajo agrario de los indígenas, en condiciones de explotación y maltrato, que a cambio reciben un minifundio o parcela de terreno para 
sembrar maíz, papas, un poco de trigo o cebada. Dentro de ese territorio "la señora de la hacienda" enseña "la doctrina cristiana" a los indígenas ¡Qué contradicción! El Dios de los pobres había sido vaciado de sentido en la práctica y discurso de la iglesia jerárquica para hacerlo funcional a la estructura económico social y despersonalizar a los indios y campesinos. Alienación lo llamaba Marx. Más aun, la connivencia entre el poder económico y la iglesia se expresaba en la edificación del templo, dentro de los límites de la hacienda, con lo que el presbítero queda prisionero del territorio simbólico-geográfico.

En este ambiente histórico-eclesial Leonidas Proaño es nombrado obispo de la provincia de Chimborazo en 1954 y comienza por conocer la realidad de la gente; en una conversación con sus sacerdotes recordaba los primeros pasos de su práctica pastoral "Yo anduve por toda la diócesis viendo quiénes eran los indios, dónde vivían, cómo vivían, y también cómo lloran los indios". Es decir, viendo y sintiendo esa realidad desde su formación teológica, pero sobre todo desde su sensibilidad humana, Proaño empieza a desarrollar una evangelización centrada en la figura del Buen Samaritano (LC. 10, 25-37) que se encontró con el "indio que había sido molido a golpes en su caminar por la historia" sí, molido a golpes por la historia de opresión de la colonia española y luego por el régimen político republicano que a semejanza de los ladrones de la parábola habían robado los bienes del caminante $y$, también a semejanza de algunos, neosacerdotes y neolevitas, religiosos que por llegar temprano al rito no se detuvieron a auxiliar la miseria de los indios, sino que muchas veces bendijeron al dueño de hacienda. Este encuentro desata una relación de recuperación y dignificación del pueblo indígena que en primera instancia encuentra en la palabra y práctica de Proaño y, progresivamente en muchos pastores y laicos comprometidos del Chimborazo, una Iglesia solidaria, sanadora, restauradora de su dignidad, que con el paso de tiempo, permitió que los indígenas se conviertan en sujeto colectivo con identidad y agenda ético-política propia.

En medio de esta emergencia pastoral renovadora, acontece el Concilio Vaticano II, que posibilita el "giro copernicano" a la teología, que entiende que la Iglesia no es la sociedad perfecta más allá del juego de la historia, sino que es el Pueblo de Dios y está llamado a ser signo de los valores del Reino. La tarea de la Iglesia según el documento conciliar Gaudium et Spes es ser solidaria con el mundo "los gozos y las esperanzas, las tristezas y las angustias de los hombres de nuestro tiempo, sobre todo de los pobres y de cuantos sufren, son los gozos y las esperanzas, tristezas y angustias de los discípulos de Cristo". Incluso en este espíritu, 
cerca de 40 pastores latinoamericanos entre los que se cuenta L. Proaño, al finalizar el Concilio firmaron el Ilamado Pacto de las Catacumbas, en la Catacumba de Santa Domitila, "pacto propuesto por Dom Helder Cámara, en quese comprometían a vivir de modo sencillo, renunciar a la riqueza, renunciar a títulos de poder, apoyar a las personas que se comprometan con los pobres y trabajadores"

En este sentido el principio articulador del primer momento de la teología de la liberación del Ecuador es la categoría solidaridad con el negado en su condición de persona, de allí que en los primeros años el discurso teológico se hablara en la Iglesia de Riobamba de hominización, es decir, humanizar al negado en su condición humana, dignificar al hombre en sentido genérico. Pero para sacar adelante la tarea humanizadora, Proaño construyó un discurso teológico sencillo, pedagógico, pastoral cargado de contenido, como tierra, comunidad, organización, educación, que constituyen al mismo tiempo las características de su teología.

En primera instancia es necesario que la comunidad indígena tenga la tierra en sus manos, que precisamente estaba en manos de los hacendados, incluso en manos de la misma Curia de Riobamba, que por cierto fue la que comenzó a devolver la tierra a sus legítimos dueños, los indios. El valor de la tierra, de la Pachamama, quizás es el primer aprendizaje que recibe de la cultura indígena Mons. Proaño, pues para el indio la tierra es fuente de vida e identidad, más aún en la sociedad agraria ecuatoriana de finales de los años 50 y comienzos de los 60, por eso se constituyó en leitmotiv de sus reflexiones, en sus homilías y en su práctica. No es extraño que sus escritos sencillos estén salpicados de citas bíblicas en torno a la tierra. Cultiva la vivencia comunitaria, tan arraigada en la cultura indígena y que es al mismo tiempo un signo fundamental de la fe cristiana, por eso, en 1977 como fruto de su vivencia y manifiesto utópico publicó el libro Creo en el hombre y la comunidad. Proaño también entendió que la fe en Jesús abre al trabajo por la justicia, más aún en una sociedad tan inequitativa, injusta y excluyente como la del Chimborazo para quienes los indios solo eran una fuerza de trabajo. Por ello, decía el Obispo," Hay que caminar con los dos pies, uno en la comunidad y otro en la organización"; no es de extrañar que desde el inicio de su tarea evangelizadora la iglesia de Riobamba empezara a tejer la organización indígena, no solo como fuerza interna, que ya la tenía, sino como fuerza para canalizar demandas sociales y políticas que se concretaron en décadas subsiguientes. Hay una característica más de esta teología, es teología popular, por eso su aliada fue la pedagogía de Paulo Freire; muy tempranamente, en 1962, Riobamba empezó a 
escuchar una nueva radio, "Escuelas Radiofónicas Populares" cuyas motivaciones para crearla definen el horizonte teológico: "Organizar campañas de alfabetización por radio y por otros medios audiovisuales. Instruir a las clases populares en las materias fundamentales para su desarrollo. Incorporar al campesino indígena a la cultura y a la vida social y económica de la nación, mediante su educación integral y estimulando su propio esfuerzo". Un libro que condensa la articulación entre fe, política y educación popular es Concienciación, Evangelización y Política, que Proaño publica en 1974.

La Teología de Proaño encarnada en la vida, había tomado partido, construir humanidad en los indígenas, dejando de lado la construcción de la Catedral, decisión que le trajo como consecuencia la antipatía de la gente urbana citadina que lo empezó a llamar "obispo de los indios" tampoco dejó indiferente a la jerarquía eclesial que veía en la pastoral del Chimborazo un brazo del comunismo ateo, por ello no es de extrañar que algún obispo preocupado por la doctrina y no de la vida del pueblo, en los años 70, denunciara a Proaño ante el Vaticano, que se hizo presente en 1973 con un Visitador Apostólico quien "durante una misa campal que celebró en el patio de las Escuelas Radiofónicas, Juanita (Males) dio este testimonio, que parece una página de los Hechos de los Apóstoles. Se acercó al Visitador. Le entregó una bufanda. "Entregarás esto, le dijo, al Papa Pablo; pero no sacarás a Taita Obispo: porque con él, ojo está aprendiendo a ver, oreja está aprendiendo a oír, boca está aprendiendo a hablar, pie nomás todavía no camina" Testimonio que conmovió al visitador y entendió el papel evangelizador de Proaño.

Este primer período profético de la teología de la liberación ecuatoriana se puede sintetizar en la misma poesía de Proaño hecha canción en los años 80.

Mantener siempre atentos los oídos/ al grito de dolor de los demás/y escuchar su pedido de socorro /es solidaridad, solidaridad, solidaridad. /Entregar por amor hasta la vida/es la prueba mayor de la amistad, les vivir y morir con Jesucristo / la solidaridad.

\section{LA TEOLOGÍA DE LA LIBERACIÓN. iLA OPCIÓN POR LOS POBRES!}

En América Latina la teología de la liberación eclosionó apoyada por el magisterio de la Iglesia, en concreto, del
Concilio Vaticano II, de la Encíclica Populorum Progressio y las Asambleas de la Iglesia Latinoamericana de Medellín 
1968 y Puebla 1979 y de la vida marcada por las luchas y esperanzas de cambio que se estaba generando en varios países del continente. Ecuador también se vio influenciado por aquel momento Kairológico, con algunas señales de identidad específicas.

Si el primer momento de la teología de la liberación en el Ecuador estuvo marcado por la hacienda como modo de producción, el segundo momento se caracteriza por dos hechos históricos: el primero, la reforma agraria que se inicia en el año 1963, como una forma de erradicar formas precarias de producción e incorporar al Ecuador al capitalismo. Este hecho está acompañado de luchas por la tierra encabezados por indígenas y campesinos que por la vía jurídica o económica buscan contar con un pedazo de tierra para sus labores agrícolas, pero rápidamente descubrieron que la tierra no era suficiente; eran necesarios otros recursos y estrategias para poder producir y mantener una economía de sobrevivencia. El segundo, el comienzo de la producción petrolera en 1972, que permitió el desarrollo de infraestructura y una incipiente industria nacional inspirada en las políticas de la CEPAL. Efectivamente, Quito y Guayaquil son las primeras ciudades beneficiarias del llamado progreso. Esta nueva realidad hizo que los indígenas y campesinos sin tierra o con tierras semiproductivas se sientan atraídos por "las luces de la ciudad", migran a los centros urbanos, pero al no tener lugar en ellas, se asientan primero en tugurios, y luego por vía de invasiones forman barrios periféricos sin servicios básicos, marcados por la pobreza y por la manipulación de políticos oportunistas que ven en los migrantes pobres "masas manipulables" de acuerdo con sus intereses.

Es en este ambiente de pobreza urbana y de luchas campesinas donde emergen nuevas experiencias eclesiales inspiradas en el magisterio latinoamericano y la praxis evangelizadora de Leonidas Proaño. Teoría y práctica eclesiales que abrieron la ruta a otras Iglesias locales. Por eso, a finales de los años 60 y comienzos de los 70 aparecen nuevos lugares teológicos inspirados por la teología de la liberación en el país. En concreto, zonas misioneras amazónicas con fuerte presencia de comunidades indígenas como Sucumbíos, con la figura de Mons. Gonzalo López Marañón, la provincia de Orellana, el Coca, donde el trabajo misionero se asocia a los Capuchinos y al nombre de Mons. Alejandro Labaka, que tendrán como reto la evangelización de comunidades indígenas en medio de los intereses de grandes empresas petroleras que ven en los pueblos indios un obstáculo para sus ambiciones. Además, en la región costera, en las provincias de Los Ríos y El Oro, el grupo misionero vasco, también inspirado por esos documentos y la práctica eclesial de la iglesia del Chimborazo, posicionó en sus escenarios geográfico-so- 
ciales un horizonte teológico liberador, Mons. Jesús Martínez fue un referente.

Luego, hacia finales de los años 70 y la década del 80, la espiritualidad liberadora se hace presente en la ciudad de Quito, sobre todo, en la zona sur donde surge un fuerte movimiento de comunidades eclesiales de base que articulan fe y vida, iglesia y compromiso social; hasta hoy está viva la presencia espiritual del padre José Carollo, de Graciano Mazón y de la Hna. Rosa Zúñiga animadores emblemáticos de "la Quito Sur". En la provincia del Azuay, se realiza un fuerte trabajo evangelizador en las comunidades campesinas y urbanas que genera el despertar ético entre grupos populares y en la misma sociedad azuaya. Las figuras de José Luis Caravias S.J., Hernán Rodas, Fernando Vega y Mons. Luna Tobar se asocian al trabajo liberador de esta provincia.

La práctica pastoral de este momento histórico tiene identidad teológica:

Posicionamiento del Dios liberador de Éxodo 3,7-14, que es capaz de ver, escuchar, sentir y comprometerse con la liberación de un pueblo esclavo al que le promete liberarlo del poder del Faraón y dar una tierra para que vivan como hermanos. En las reuniones de centenares de CEB, los textos bíblicos donde aparece la figura del Dios liberador, son leídos y releídos en la Biblia latinoamericana, pues, encuentran en Yavé la figura simbólica de la justicia y en el faraón la figura diabólica del poder político-económico generador de injusticia.

Se descubre la figura del Jesús histórico, humano, cercano a las necesidades y aspiraciones del pueblo pobre, pero sobre todo la centralidad de Reino de Dios, como don y tarea a construir. No cabe ya el discurso religioso de "salvar el alma" por medio de la confesión del credo y la participación en la liturgia, es necesario trabajar apasionadamente por hacer presente entre nosotros el Reino de justicia y paz y el resto vendrá por añadidura. Por ello, se supera la dicotomía entre lo espiritual y lo material, entre lo religioso y lo político, superando también esa equivocada interpretación de la expresión "Mi reino no es de este mundo", pues el Reino de Dios irrumpe en el mundo, en la sociedad con la presencia liberadora de Jesús, dando vista a los ciegos, libertad a los cautivos, y anunciando el año de gracia del Señor (LC. 4, 16-20).

Surge un nuevo modo de ser Iglesia inspirado en el documento conciliar Lumen Gentium, que comprende a la comunidad cristiana como Pueblo de Dios, con la tarea fundamental de ser signo y fermento del Reino. Ese nuevo rostro de Iglesia tiene nombre propio, Comunidades Eclesiales de Base, campesinas, indí- 
genas, urbanas, populares, cuyos miembros son pobres y gente comprometida con la causa de los pobres, unidos por la fe en Jesucristo, sus necesidades comunes y su ideal de vivir como hermanos en una sociedad que se la sueña más justa y solidaria. Es una Iglesia viva, ministerial, con animadores comunitarios que frecuentemente son personas sencillas con poca educación formal pero con gran sabiduría vital y vivencia de fe. Animadores de la pastoral de la salud en zonas donde muchas veces el Estado está ausente y la Iglesia, desde la solidaridad y la autogestión comunitaria crea boticas populares y acompaña a los hermanos enfermos. Ministros de la solidaridad, que desde el compartir y la creatividad apoyan a las personas y familias más necesitadas de la comunidad. Cantores y músicos que en las celebraciones expresan estéticamente su fe. Educadores populares que concientizan a la comunidad de lo que acontece en la compleja realidad. Incluso en algunas comunidades más vulnerables surgen defensores de derechos humanos y ministros que impulsan la autogestión comunitaria.

Las CEB, alimentan su espiritualidad liberadora en reuniones semanales donde acompañadas por un animador o animadora refuerzan su compromiso con el Reino de Dios, y en su barrio celebran, cantan y comparten un café. En las reuniones la biblia es leída, fijándose más en el espíritu del texto que en la letra, para descubrir el plan de Dios y despertar la conciencia solidaria de la comunidad. Además se cuenta con guías bíblicas como Vivir como hermanos y Cristo Compañero de J. Luis Caravias, Círculos bíblicos de Carlos Mesters, y un sinnúmero de folletos escritos por agentes de pastoral con claridad pedagógica y lenguaje sencillo que sirven de pauta para ver la realidad de pobreza, juzgar la realidad a la luz de las Escrituras, es decir, descubrir la novedad de Jesús, los ideales y problemas de las comunidades neotestamentarias y asumir pequeños compromisos comunitarios.

Este espíritu y teología, se fortalecen en los encuentros anuales de las CEB, en donde agentes de pastoral y miembros de comunidades de las iglesias del Chimborazo, Sucumbíos, Coca, Quito Sur, Azuay, Los Ríos, El Oro, entre otras, hacen análisis de coyuntura, comparten el caminar de sus iglesias locales y delinean compromisos comunes. Junto a esta práctica, son emblemáticos los equipos de reflexión de comunidades de Vida Religiosa inserta en medios populares (CRIMPO), que se convirtieron en espacios de revisión de vida y de producción teológica.

La teología de la liberación, no solo que inspira un conjunto de convicciones y prácticas de renovación intraeclesial, sino que desde dentro de las 
comunidades generan un nuevo tejido social liberador como: organizaciones barriales que buscan mejorar las condiciones de vida, comités de solidaridad con Centroamérica que fueron significativos en las ciudades de Quito, Cuenca, Riobamba, Lago Agrio; organizaciones de derechos humanos como la Comisión Ecuménica de Derechos Humanos que se comprometen con la práctica de denuncia y defensa de los derechos de las personas; organizaciones indígenas nacidas y animadas por Proaño, López Marañón, misioneros capuchinos, misioneros salesianos y desde luego, por el trabajo de comunidades religiosas femeninas como Lauritas, Esclavas del Sagrado Corazón entre otras; organizaciones de educación popular, que generan procesos que permiten pasar de una conciencia mágica o ingenua a una conciencia crítica propuesta por Paulo Freire y acogida en centros de formación como Santa Cruz Riobamba o la parroquia Cristo Resucitado de Quito, donde permanentemente llegan educadores, teólogos, biblistas que aportan en la toma de conciencia de la realidad, el cultivo de la espiritualidad Reino-céntrica y el compromiso con la historia.

A nivel jerárquico eclesial, este tiempo es de sensibilidad de algunos pastores que movidos por los documentos fundantes del Magisterio de la Iglesia latinoamericana como Medellín y Puebla, permiten una renovación teológica de los agentes de pastoral e invitan a un compromiso menos ritual y más solidario con los pobres. De este sector eclesial surgieron varias organizaciones, sobre todo ONGs que impulsan programas y proyectos de desarrollo para los pobres.

Otros obispos desde una intencionalidad incluso no muy entendida por ellos mismos, pero apelando a que la Iglesia es de todos, promovieron la emergencia de nuevos movimientos eclesiales de tipo pentecostal, que desde un cierto dualismo de cuerpo-alma, santopecador, iglesia-mundo formaron grupos altamente emotivos, donde la acción del Espíritu Santo se confunde con el nivel de mayor o menor emotividad de las personas, que se reconocen pecadores individualmente pero no reconocen las estructuras de pecado. Sociológicamente, los líderes de este movimiento, por lo regular son comerciantes, movidos por la teología de la bendición y políticamente alineados a sectores conservadores, por eso en época de elecciones pedían a sus miembros votar por los candidatos de partidos tradicionales. También auspiciaron movimientos "liturgocéntricos", que usando recursos altamente afectivos logran cohesionar grupos cuya preocupación fundamental es la celebración litúrgica y el estudio literal de la biblia. Sin duda, estos y otros movimientos emotivos y biblio-céntricos son críticos de la teología de la liberación; por eso, en sus reuniones es muy común es- 
cuchar aclaraciones sobre la opción preferencial por los pobres proclamada por la Iglesia en las Conferencias de Medellín y Puebla, con expresiones como "la opción de la Iglesia es por los pobres, pero no solo por los pobres materiales sino también por los pobres espirituales".

Un tercer sector muy conservador ligado a intereses económicos y de prestigio, no toleró la teología de la liberación y la combatió desde sus espacios, acusando a los obispos como Leonidas Proaño de comunista. Impulsaron, o al menos toleraron, a la organización Tradición, Familia y Propiedad, que en la siguiente época de la TLE se transformará en "Heraldos del Evangelio". Desde luego, también auspiciaron el fortalecimiento de la Prelatura del Opus Dei y la creación de nuevas CEB de carácter emotivo-ritual en sectores populares y campesinos.

En los años 80, particularmente entre 1984 y 1988 desde el Estado se realizó una persecución sistemática a laicas -laicos, religiosas- religiosos comprometidos con los pobres, que eran vistos por el gobierno de turno como grupos de comunistas infiltrados en la Iglesia. Elina Guarderas, recordando la presencia de la mujer en la iglesia y la sociedad dice:

En Cotopaxi como CRIMPO logramos el respeto, que no nos vieran como las "monjitas", sino como mujeres comprometidas con el pueblo, y se logró mucho, desgraciadamente duró poco tiempo porque tuvimos que salir por distintas circunstancias, algunas expulsadas por los gobiernos, por la represión militar, Alma Montoya fue tomada presa la había cogido la Brigada Patria y yo fui amenazada de muerte, el gobierno fue muy represor, yo tuve que salir, viví una de las dificultades a nivel espiritual terriblemente grande y dolorosa, me sentí como el asalariado que deja a las ovejas ahí y huye.

La TLE por medio de sus figuras emblemáticas crea una atmósfera que invita al compromiso con los más pobres, con la justicia, con la liberación del país. Esa atmósfera cuenta con lugares geográficos concretos, donde se vive y sueña con el Reino histórico y hace que jóvenes, intelectuales, obreros, organizaciones y sectores críticos de la sociedad tiendan puentes de diálogo con esta nueva Iglesia. No es casualidad que en ciudades y provincias que antes eran muy conservadoras, por la presencia de una práctica pastoral liberadora, empezaran a legitimar discursos y opciones político-sociales más comprometidas con los sectores populares.

En síntesis la teología liberadora del Ecuador en este segundo momento posiciona al pobre social y económico como sujeto histórico, capaz no solo de ser objeto de la bondad paternalista de la Iglesia sino de ser actor que camina movido por la utopía del Reino anunciado por Jesús de Nazaret. Por eso, en 
casi todas las CEB es común escuchar la canción "Un pueblo que camina por el mundo/ gritando ven Señor. / Un pueblo que busca en esta vida/la gran liberación/.
Los pobres siempre esperan el amanecer/ de un día más justo y sin opresión/los pobres hemos puesto la esperanza en ti/libertador".

\section{LAS TEOLOGÍAS DE LIBERACIÓN ¿LA OPCIÓN POR LAS VÍCTIMAS!}

La caída del socialismo real, la pérdida electoral del movimiento sandinista en Nicaragua y el triunfo económico del Consenso de Washington con su agenda neoliberal, fueron acontecimientos externos que al parecer dejaba sin piso histórico viable a la luchas enmancipatorias de los pueblos de América Latina. No hay alternativas, el futuro es más de lo mismo se escuchaba con frecuencia en la voz de los ideólogos criollos del neoliberalismo. En este marco internacional de utopías ausentes, la teología de la liberación sufrió un revés que amenazaba su supervivencia como reflexión crítica desde la fe. Inclusive algunas comunidades religiosas y misioneros se desencantaron de América Latina y salieron de sus lugares de evangelización. Sin embargo, como la realidad de pobreza y exclusión se agudizó en esta geografía, la teología de la liberación tercamente sigue su fidelidad al Reino, porque mientras los sistemas generen pobreza y exclusión y haya comunidades de fe que reflexionen sobre su realidad seguirá existiendo teología de la liberación. Además, la TL en este período incorpora nuevos actores, nuevas hermenéuticas, nuevas herramientas de análisis como la antropología cultural y replantea los horizontes de liberación más contextuales.

A nivel nacional, como fruto de la aplicación de las políticas neoliberales, la disminución del tamaño del Estado y la desinversión en las áreas de educación, salud y vivienda la pobreza crece. En los años noventa, Ecuador aparece con un 60\% de su población en condiciones de pobreza. Como consecuencia, muchos migran a los países del norte como Estados Unidos y España. La deuda externa se convierte en el peor obstáculo para el desarrollo humano, es más, se convierte en el modo de garantizar la dependencia del país frente al capital financiero representado por los organismos internacionales de crédito como el FMl y Banco Mundial. Además, en medio de la crisis emergen nuevos actores sociales con demandas locales, regionales y globales y postulan la resistencia al neoliberalismo.

Pastores, religiosos y teólogos, sensibles a la nueva realidad entienden que esta se constituye en el lugar teológico desde el cual Dios habla, se reflexiona 
comunitariamente la vida desde la fe y se construye la nueva savia de la teología de la liberación. En los años 90 se dan encuentros, reuniones, cursos, pronunciamientos para entender desde la fe, la misión de la iglesia en estas nuevas circunstancias.

Los principios que guían la teología y práctica evangelizadora de la liberación, en este nuevo período, son resistencia, vida y diversidad. La Resistencia a los nuevos proyectos de globalización de la economía, que se alimenta desde la experiencia bíblica de los Macabeos, que frente a la invasión cultural griega resisten apelando a sus valores religiosos y desde la cultura profunda que inspira la vivencia y práctica los pueblos y comunidades. El principio vida cobra sentido, pues frente a los proyectos de muerte que empobrecen, excluyen, y poco a poco matan no solo la vida individual sino colectiva de comunidades, se posiciona el Dios apasionado de la vida. Al mismo tiempo se descubre la urgencia del cuidado de la vida de la gran oikos. El principio diversidad, emerge porque no basta con identificar a los pobres, estos pobres tienen rostro propio, indígenas, afroecuatorianos, campesinos, pobladores, mujeres, migrantes, que son víctimas de la exclusión, y que al mismo tiempo, reclaman participación en la sociedad y en la Iglesia. Es decir, se construye una teología con hermenéuticas específicas.
Los rasgos de identidad de la teología liberadora son los siguientes:

- Es una teología que incorpora lo étnico, que no era la preocupación principal en la fase anterior, pero que dada la nueva realidad fue objeto de tematización desde la teología, en muchos casos, desde la categoría "semillas del Verbo", y otras cercanas como inserción, inculturación, encarnación e interculturalidad pasan a ser palabras clave para la comprensión del nuevo momento teológico. Cerca del quinto centenario del "descubrimiento" de América, la reflexión histórico-teológica va por deslegitimar la celebración de los 500 años de conquista y colonización, donde nuevamente el movimiento indígena es protagonista, pues afirma que no hay nada que celebrar, por el contrario, consideran que es tiempo de recordar 500 años de resistencia y de proyectar la agenda social para las nuevas décadas. En 1990 sucede un acontecimiento histórico, el levantamiento indígena, acontecimiento que tiene que ser leído simbólicamente, pues es el acto de mayor trascendencia político-cultural que realiza el movimiento. Es su visibilización pública como indios, no para mendigar al poder sino para exigir sus derechos frente al Estado, no para conquistar el poder sino como forma de construir el poder.

- En esta misma línea se ubica la reflexión teológica realizada en las co- 
munidades afro-descendientes, fruto de un largo proceso de evangelización y dignificación realizado por los misioneros combonianos, quienes desde su opción de acompañar la evangelización de las comunidades negras, sobre todo en la provincia de Esmeraldas, descubren en las páginas de la biblia la presencia y ausencia de los negros y en diálogo entre el pasado y el presente, de cultura a cultura, de fe a fe, comprenden la misión de los pueblos afroecuatorianos hoy.

- Es una teología de la liberación con rostro de mujer que, en primer término, en la primera y segunda fase de la TLE son las protagonistas de las comunidades eclesiales, las que forman parte de equipos pastorales, las que animan, las que cantan en la liturgia, las que tienen a su cargo ministerios de la caridad y la catequesis, pero, no obstante, son las que sufren el peso de las estructuras patriarcales de la sociedad y a veces en la misma Iglesia, por eso, en la tercera fase son las mismas mujeres las que desde su propia mirada y lectura de las fuentes de la teología sistematizan su teología y la escriben. Desde su visión teológica y con la agenda liberadora empiezan a realizar encuentros, cursos de formación, reuniones en donde conversan sobre su situación de mujer, visibilizan las formas patriarcales de dominación del varón en la casa y la sociedad. Leen la biblia desde su condición, visibilizan el rostro ma- terno de Dios, rescatan el protagonismo de las mujeres dentro de la historia de la salvación. Es más, afirman que la práctica y palabra de Jesús es el canon para interpretar los textos tanto del primer como segundo testamentos, sobre todo de las cartas paulinas que si no se hace una lectura Cristo-céntrica de ellas, se puede justificar y reforzar formas patriarcales de organización social y eclesial. Además, se incluyen los talleres y ceremonias rituales para restaurar a las mujeres víctimas de la sociedad androcéntrica.

Según Margarita María Pinos una de las contribuciones del momento actual de la reflexión teológica feminista en América Latina, "es la recuperación de una cosmovisión holística donde la humanidad se descubre como parte de un todo, integrada a la energía vital de la naturaleza. El eco feminismo reafirma elementos fundamentales de las teologías ancestrales indígenas." Es así que en el Taller que realizan las mujeres en Riobamba, inician con un ritual a través del que expresan su resistencia a la discriminación y exclusión como etnia y como mujeres, alli se resignifican y se recrean.

Es una ecoteología del cuidado de la vida en todas sus formas. Esta emerge sobre todo entre agentes pastorales de la cuenca amazónica, de las provincias de Orellana y Sucumbíos, zona altamente biodiversa, donde habitan pueblos profundos en autoaislamiento, pero que al mismo tiempo es la fuente de explotación petrolera que causa la contamina- 
ción del suelo, del agua y atenta contra el frágil equilibrio ecológico amazónico. Desde finales del siglo XX y comienzos del XXI, se cultiva una teología y espiritualidad de la creación, del Dios de la vida, presente en todas las formas de vida. En la misión capuchina de la Amazonía se busca vivir"la relación fraterna entre todos los seres, porque antes de pensar en Dios, la realidad nos descubre y demuestra que todos los seres estamos conectados, por eso debemos cultivar una espiritualidad de la fraternidad entre los seres". El texto de Génesis dos, que narra la relación armónica entre la humanidad y la naturaleza y define la misión del jardinero en la frase"cultivar y cuidar el huerto" $(G n 2,15)$ es inspirador para la tarea evangelizadora y para la espiritualidad de las comunidades. Esta teología ha permitido el diálogo con los nuevos actores y con las organizaciones sociales. Hay que recordar que en fase anterior de la TLE, monseñor Labaka y la hermana Inés Arango por la defensa de los pueblos que habitan en la gran oikos amazónica paradójicamente fueron lanceados por un grupo de waoranis que defendían su casa.

Esta práctica pastoral liberadora que en fidelidad a Jesús optó por unos sujetos concretos, modeló un nuevo tipo de organización eclesial y creó también un nuevo modo de hacer reflexión teológica, recibió la censura al interior de la jerarquía católica, la misma que se visibilizó en la "intervención" del Vaticano en la Iglesia de Sucumbíos. Aceptó la renuncia de Mons. Gonzalo López el 30 de octubre de 2010 por límite de edad, y para borrar todo el trabajo liberador nombró como Administrador Apostólico al P. Rafael Ibarguren Schindler, bajo el argumento que "La visión pastoral llevada adelante por usted (Mons. Gonzalo) no siempre era conforme con la exigencia pastoral de la Iglesia" por lo que "el nuevo Administrador Apostólico tendrá que organizar el Vicariato e implantar de manera diferente todo el trabajo pastoral"

Asimismo, algunos líderes de las CEB y organizaciones eclesiales fuertes en la segunda época, en las década de los noventa y comienzos del siglo XXI, frente a la urgencia de atender las necesidades básicas de cuidado infantil, educación, salud, constituyeron ONGs de asistencia social, lo que en primer lugar es una respuesta inmediata en favor de la población, "dar de comer al hambriento y de beber al sediento", pero luego, desmoviliza a las CEB que pierden capacidad de articulación en función del horizonte teológico liberador y se reducen a ser administradores de los pequeños proyectos de subsistencia, línea teológica que hoy también ha cobrado fuerza en la reflexión de la teología del desarrollo, la cooperación internacional, etc.

Sin embargo, las teologías de liberación desde rostros y hermenéuticas específicas, ha hecho que las organizacio- 
nes eclesiales laicales sigan fortaleciendo la interlocución con organizaciones feministas, étnicas, ecologistas, juveniles, alterglobalizadoras que descubren en la espiritualidad liberadora unos valores fundamentales que inspiran sus luchas y aportan para la construcción de la agenda ético-social para nuevas décadas, siempre mirando al frente y en fidelidad a los excluidos.

En conclusión, la teología de la liberación ecuatoriana en sus tres etapas sin duda que encuentra en Leonidas
Proaño el referente de mayor significación e inspiración. Tiene como fortaleza ser vivida, hablada y escrita por pastores, religiosos y teólogos que caminan con las comunidades. Es una teología reinocéntrica, que busca la dignificación de la persona, cultiva la solidaria entre los pobre. Está comprometida con rostros específicos e inspira las prácticas de defensa y cuidado del ambiente. Estos principios, para alegría de las CEB, están presentes en el discurso y espiritualidad del Papa Francisco, que sin duda ha rehabilitado la Teología Latinoamérica. 
Revista PUCE, ISSN 1012-389X. Núm, 102

\section{BIBLIOGRAFÍA}

Aciprensa. (10 de febrero de 2013). El Papa nombra administrador apostólico para Sucumbíos Ecuador. Aciprensa, pág. 1.

Concilio Vaticano II. (1965). Gaudium et Spes. Vaticano : BAC.

Educación, M. d. (1989). Monseñor Proaño. Su mensaje en anécdotas. Documento de trabajo 7. Campaña Nacional de Alfabetización Monseñor Leonidas Proaño. Quito: Ministerio de Educación.

García, J. (15 de Mayo de 2015). La teologia de la liberación en la misión capuchina de Orellana (B. Tobar, Entrevistador).

Moreno, J. (1987). La Iglesia en Ecuador. Riobamba (desde 1962). En CEHILA. Sígueme 1987. En E. Dussel, Historia General de la Iglesia en América Latina VIII. Perú, Bolivia y Ecuador. Salamanca: Sígueme.
Obispos, reunidos en el Vaticano II. (1965). El pacto de las Catacumbas . (pág. 2). Vaticano: Iglesia. cl Conferencia Epispocal de Chile.

Pineda, C. (2013). Tesis maestría "Mujeres y teología de la liberación en Riobamba y Quito: los decenios de 1970 y 1980". Programa de maestría en historia. Quito: Universidad Andina.

Vera, A. S. (4 de mayo de 2015). La práctica pastoral de Mons. Proaño (B. T. Solano, Entrevistador). 